\title{
A bibliometric analysis of natural language processing in medical research
}

\author{
Xieling Chen ${ }^{1}$, Haoran Xie ${ }^{2}$, Fu Lee Wang ${ }^{3}$, Ziqing Liü ${ }^{4}$ Juan $\mathrm{Xu}^{5}$ and Tianyong Hao ${ }^{6,7^{*}}$ \\ From The 3rd China Health Information Processing Conference \\ Shenzhen, China. 24-25 November 2017
}

\begin{abstract}
Background: Natural language processing (NLP) has become an increasingly significant role in advancing medicine. Rich research achievements of NLP methods and applications for medical information processing are available. It is of great significance to conduct a deep analysis to understand the recent development of NLP-empowered medical research field. However, limited study examining the research status of this field could be found. Therefore, this study aims to quantitatively assess the academic output of NLP in medical research field.
\end{abstract}

Methods: We conducted a bibliometric analysis on NLP-empowered medical research publications retrieved from PubMed in the period 2007-2016. The analysis focused on three aspects. Firstly, the literature distribution characteristics were obtained with a statistics analysis method. Secondly, a network analysis method was used to reveal scientific collaboration relations. Finally, thematic discovery and evolution was reflected using an affinity propagation clustering method.

Results: There were 1405 NLP-empowered medical research publications published during the 10 years with an average annual growth rate of 18.39\%. 10 most productive publication sources together contributed more than 50\% of the total publications. The USA had the highest number of publications. A moderately significant correlation between country's publications and GDP per capita was revealed. Denny, Joshua C was the most productive author. Mayo Clinic was the most productive affiliation. The annual co-affiliation and co-country rates reached $64.04 \%$ and $15.79 \%$ in 2016, respectively. 10 main great thematic areas were identified including Computational biology, Terminology mining, Information extraction, Text classification, Social medium as data source, Information retrieval, etc.

Conclusions: A bibliometric analysis of NLP-empowered medical research publications for uncovering the recent research status is presented. The results can assist relevant researchers, especially newcomers in understanding the research development systematically, seeking scientific cooperation partners, optimizing research topic choices and monitoring new scientific or technological activities.

Keywords: Natural language processing, Medical, Bibliometrics, Statistical characteristics, Scientific collaboration, Thematic discovery and evolution

\footnotetext{
* Correspondence: haoty@gdufs.edu.cn

${ }^{6}$ School of Information Science and Technology, Guangdong University of Foreign Studies, Guangzhou, China

${ }^{7}$ School of Computer, South China Normal University, Guangzhou, China

Full list of author information is available at the end of the article
} 


\section{Background}

Natural language processing (NLP) is a theoretically motivated range of computational techniques for the automatic analysis and representation of human language [1]. Its goal is to realize human-like language understanding for a wide range of applications and tasks [2]. As a large and complex domain, medicine is rich in synonymy and semantically similar and related concepts [3]. Most clinical information resources including Electronic Medical Records (EMRs), Electronic Health Records (EHRs) and medical knowledge contain considerable amount of information. However, much of this information comes in unstructured form, also called free-text [4]. NLP is crucial for transforming relevant unstructured information hidden in free-text into structured information and is extremely useful in improving healthcare and advancing medicine [5].

There have been rich research achievements of NLP methods and applications for processing medical information [6]. Emerging interests of medical information processing with NLP methods include speech information recognition [7], semantic labeling [8], syntactic parsing [9], word sense disambiguation [10, 11], negation detection [12], and temporal analysis [13, 14]. Medical practical problems can also gain solutions from NLPempowered applications including adverse drug reactions detection [15], medication discrepancy detection [16], EMRs or EHRs coding and classification [17], clinical trial computation [18-21], etc. NLP-empowered medical research field grows fast and draws more and more attention [6]. It is of great significance to understand its research status through a systematic analysis on relevant research output.

In the analysis research, bibliometrics is defined as the use of statistical methods for quantitative assessment of academic output [22, 23]. Benefits of bibliometric analysis include evaluating leading scientific researchers or publications [24], studying the structure of the network of a scientific field [25], identifying major topics [26], discovering new developments [27], etc.

This paper thus carries out a thorough bibliometric analysis on NLP-empowered medical publications from PubMed during the year 2007-2016. The descriptive statistics analysis, social network analysis, and Affinity Propagation clustering analysis are used in the analysis. Specifically, the purpose of the analysis is to: 1) identify productive publication sources, authors, affiliations, and countries in NLP-empowered medical research field; 2) visualize the number of countries and scientific collaboration among authors and affiliations; and 3) distinguish major themes and their evolution.

\section{Related work}

Applications of bibliometrics are numerous. Many studies focused on publication statistical characteristics evaluation with elements such as publication data, influential journals, productive authors, affiliations, and countries. Based on two separate databases Web of Science (WoS) and Google Scholar, Diem and Stefan [28] investigated the fitness-forpurpose of bibliometric indicators for measuring the research performance of individual researchers in education sciences field in Switzerland. The study results indicated that the indicators for research performance measurement such as quantity of publications and citation impact measure were highly positively correlated. Fan et al. [29] conducted a bibliometric study for the evaluation of the quantity and quality of Chinese publications on burns at both the international and domestic levels with basis of PubMed records during 1985 and 2014. Similar works have also been conducted for medical research output. A study for the determination of whether a correlation existed between bibliometrics and National Institutes of Health (NIH) funding data among academic neurosurgeons was conducted by Venable et al. [30]. Their work revealed that bibliometric indices were higher among neurosurgeons with NIH funding, but only the contemporary h-index was shown to be predictive of NIH funding. By examining the growth of published literature on diabetes in three countries including Nigeria, Argentina and Thailand, Harande and Alhaji [31] showed that the literature of the disease grew and spread very widely. Ramos [32] found that the research output in countries with more estimated cases of tuberculosis was less compared with industrialized countries through a bibliometric analysis of tuberculosis research output. In addition, bibliometric analysis on research publications related with cancer [33], eye disease [34], obesity [35], dental traumatology [36], etc., could also be found. Bibliometric analysis for publication statistical characteristics evaluation was also available for specific journals, e.g., Journal of Intellectual Property Rights [37] and The Electronic Library [38].

Studies on collaboration relationship among authors, affiliations, or countries were commonly found. Based on researches covering biomedical, physics, and mathematics, Newman [39] compared the scientific coauthorship patterns using network analysis. Radev et al. [40] investigated the publications published by The Association for Computational Linguistics using citation and collaboration network analysis to identify the most central papers and authors. A bibliometric and visual study on consumer behavior research publications from 1966 to 2008 was presented by Muñoz-Leiva et al. [41]. Geaney et al. [42] provided a detailed evaluation of type 2 diabetes mellitus research output during the year 1951-2012 with methods of large-scale data analysis, bibliometric indicators, and density-equalizing mapping. They came to the conclusion that the number of research was rising in step with the increasing global burden of the disease. With a chord diagram of the 20 most 
productive countries, Li et al. [43] confirmed the predominance of the USA in international geo-ontology research collaboration. They also found that the international cooperation of countries such as Sweden, Switzerland, and New Zealand were relatively high although with fewer publications.

There were also a few studies centering on research topic detection of a certain field using bibliometrics. For example, Heo et al. [44] analyzed the field of bioinformatics using a multi-faceted topic modeling method. By combining performance analysis and science mapping, some studies conducted thematic evolution detection and visualization of a given research field, e.g., hydropower [45], neuroscience [46], and social work [47]. Similar works have also been conducted for specific journals such as Knowledge-Based Systems [22]. Based on co-word analysis, Cobo et al. [48] proposed an automatic approach with the combination of performance analysis and science mapping to show the conceptual evolution of intelligent transportation systems research field during three consecutive periods. Six main thematic areas were identified out. With the purpose of mapping and analyzing the structure and evolution of the scientific literature on gender differences in higher education and science, Dehdarirad et al. [49] applied co-word analysis to identify main concepts, used hierarchical cluster analysis to cluster the keywords, and created a strategic diagram to analyze trends.

Most relevant studies chose WoS as publication retrieval data source, and therefore, author-defined keywords and ISI keywords plus were usually used as topic candidates $[22,23,46]$. This might lead to information loss without considering title and abstract fields. The key terms in title and abstract fields were extracted and analyzed using VOSviewer with equal importance in the study of Yeung et al. [46]. However, it is more reasonable to bestow weighing for terms from different fields.

To our knowledge, there was no study applying bibliometrics to assess research output of NLP-empowered medical research field. Therefore, giving the deficiencies in existing research, this study uses PubMed as data source. With 1405 NLP-empowered medical research publications retrieved, literature distribution characteristics and scientific collaboration are acquired using a descriptive statistics method and a social network analysis method, respectively. In addition to author defined keywords and PubMed medical subject headings (MeSH), key terms extracted from title and abstract fields using a developed Python program are also included in AP clustering analysis for thematic discovery and evolution.

\section{Methods}

\section{Data set}

PubMed is an important data source on life sciences and biomedical topics. We used PubMed as data source and downloaded documents using the following query: ((“2007"[Publication Date]: “2016”[Publication Date])) AND (("NLP"[Title] OR "Natural Language Processing"[Title]) OR ("NLP"[Title/Abstract] OR "Natural Language Processing"[Title/Abstract])).

Using the query, we retrieved a total of 1776 documents in XML format. Key elements including title, published year, publication source, author address, author keywords, PubMed MeSH, and abstract were extracted. Due to the issues of information missing and irrelevant documents, manual information supplement and document exclusion were conducted. After that, 1405 NLP-empowered medical research publications between 2007 and 2016 were identified out as dataset.

According to the author addresses information, the corresponding affiliations and countries were manually preprocessed and automated identified. As for the title and abstract fields, a developed Python program was applied to extract key terms (including single words and phrases). According to observation on 50 samples, we found that most of the extracted single words were meaningful, e.g., "influenza", "surveillance", and "misdiagnoses". Furthermore, in order to improve data quality, a de-duplicating process was applied (author defined keywords, PubMed MeSH, and extracted key terms as units of analysis). Some abbreviations were replaced by the corresponding full-names. For example, "EHR" was replaced by "Electronic Health Record". Words representing the same concepts were grouped. In addition, words with a very broad and general meaning, e.g., "natural language processing", "algorithm", were removed. After the above pre-processing, the dataset was analyzed using software R. Some statistical characteristics of the dataset are shown as Table 1.

\section{Statistical analysis}

Some publication characteristics are obtained through statistical analysis using a descriptive statistical method, a regression analysis method, and a hypothesis testing method. Descriptive statistics is used for quantitatively summarizing the basic characteristics of a collection of data [50]. It simplifies large amounts of data in a sensible way by presenting quantitative descriptions in a manageable form, generally along with simple graphics analysis. Regression analysis is a set of statistical processes for estimating the relationships between a dependent variable and one or more independent variables. It helps one find out how the dependent variable changes when any one of the independent variables is varied while the other independent variables remain fixed. As a method of statistical inference, statistical hypothesis testing is used to determine whether a hypothesis is a reasonable statement and should not be 
Table 1 The statistical characteristics of the dataset

\begin{tabular}{|c|c|}
\hline Characteristics & Statistics \\
\hline Total \#pub. & 1405 \\
\hline \#pub. with author address information & 1386 \\
\hline \#pub. with abstract & 1382 \\
\hline \#pub. with author keywords or PubMed MeSH & 1277 \\
\hline \#unique publication sources & 324 \\
\hline \#unique countries/first countries & $56 / 45$ \\
\hline \#unique authors/first authors & $4391 / 1053$ \\
\hline \#unique affiliations/first affiliations & $961 / 514$ \\
\hline Average \#words/word characters in title & $12.53 ; 6.50$ \\
\hline Average number/standard deviation of character in title & $95.43 ; 29.72$ \\
\hline Average \#words/word characters in abstract & $215.24 ; 5.62$ \\
\hline Average number/standard deviation of character in abstract & $1456.95 ; 536.2$ \\
\hline $\begin{array}{l}\text { Top } 10 \text { frequency words/phrases in author keywords or } \\
\text { PubMed MeSH }\end{array}$ & $\begin{array}{l}\text { Electronic health record (363; } 25.84 \%) \text {; Data mining }(278 ; 19.79 \%) ; \text { Information } \\
\text { storage and retrieval }(239 ; 17.01 \%) ; \text { Artificial intelligence }(179 ; 12.74 \%) ; \text { Female } \\
(163 ; 11.60 \%) ; \text { Semantics }(156 ; 11.10 \%) ; \text { Male }(153 ; 10.89 \%) ; \text { Controlled vocabulary } \\
(140 ; 9.96 \%) ; \text { Automatic pattern recognition }(127 ; 9.04 \%) ; \text { Medical record system } \\
(112 ; 7.97 \%)\end{array}$ \\
\hline Top 10 frequency words/phrases extracted from title & 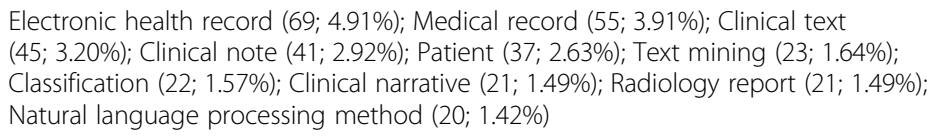 \\
\hline Top 10 frequency words/phrases extracted from abstract & $\begin{array}{l}\text { Patient (322; 22.92\%); Precision (217; 15.44\%); F-measure (205; 14.59\%); Recall } \\
\text { (178; } 12.67 \%) ; \text { Accuracy }(164 ; 11.67 \%) \text {; Electronic health record }(161 ; 11.46 \%) ; \\
\text { Natural language processing method (155; } 11.03 \%) ; \text { Medical record } \\
(143 ; 10.18 \%) ; \text { Disease }(141 ; 10.04 \%) ; \text { Concept }(128 ; 9.11 \%)\end{array}$ \\
\hline
\end{tabular}

rejected or if it is an unreasonable statement and should be rejected based on sample statistics and probability theory. A hypothesis is proposed for the statistical relationship between two datasets as an alternative, and is compared with an idealized null hypothesis proposing no relationship between two datasets. The comparison is regarded statistically significant if the null hypothesis is unlikely to realize according to a threshold probability, i.e., a significance level.

In this study, a descriptive statistics method was applied to acquire the distribution characteristics of the dataset, including publication distribution by year, productive publication sources, authors, affiliations, and countries, as well as annual cooperation publication distribution. Based on the number of publications, 3 fitting models including linear model without intercept, linear model with intercept, and non-linear model with quadratic term, were built with year/ 1000 and $(\text { year } / 1000)^{2}$ as independent variables. Akaike Information Criterion (AIC) and adjusted Rsquared $\left(\overline{R^{2}}\right)$ were used to select the optimal fitting model. In order to understand the relationship between number of publications and GDP per capita, a Spearman's rank correlation test was applied to test the hypothesis as:
Hypothesis: There is no significant relationship between publications and GDP per capita

Spearman's rank correlation coefficient is a nonparametric measure of statistical dependence between the rankings of two variables, which is expressed as:

$$
r_{s}=\rho_{r g_{X}, r g_{Y}}=\frac{\operatorname{cov}\left(r g_{X}, r g_{Y}\right)}{\sigma_{r g_{X}} \sigma_{r g_{Y}}}
$$

where $\rho$ denotes the usual Pearson correlation coefficient, but applies to the rank variables. $\operatorname{cov}\left(r g_{X}, r g_{Y}\right)$ is the covariance of the rank variables. $\sigma_{r g_{X}}$ and $\sigma_{r g_{Y}}$ are the standard deviations of rank variables.

\section{Geographic visualization}

Geographic visualization is a set of techniques for analyzing spatial data with an emphasis on knowledge construction over knowledge storage or information transmission. Aiming at facilitating the exploration, analysis, synthesis, and presentation of georeferenced information, geographic visualization integrates principles from geographic information systems, exploratory data analysis, cartography, as well as information visualization [51].Techniques such as multimedia, image processing, computer graphics, and virtual reality are combined for 
presenting information in a way that patterns can be found, and greater understanding can be acquired. In this study, we applied geographic visualization analysis to explore worldwide geographical distribution of NLPempowered medical research publications in countrylevel.

\section{Social network analysis}

Social network analysis, related to network theory, is a process of investigating social structures based on networks and graph theory in modern sociology [52]. Social network perspective concentrates on relationships among social entities [53] with two main focuses, i.e., the actors and the relationships between them in a specific social context [54]. Networked structures are characterized in terms of nodes with the ties, edges, or links connecting them.

In this study, we applied social network analysis to explore the cooperation relationships for specific authors and affiliations in NLP-empowered medical research field. The cooperation among affiliations and authors were visualized with force directed network graphs, respectively. In the network graphs, the nodes represented specific affiliations or authors, and the lines represented the cooperation relationship. The size of node indicated the number of publications of a specific author or affiliation. The width of link indicated the cooperation frequencies between the two affiliations or authors.

\section{Term importance weighting}

In thematic evolution discovery, author defined keywords, PubMed $\mathrm{MeSH}$, and key terms extracted from title and abstract were jointly used as units of analysis. Since the importance of different parts of a publication was different, we conducted a weighting process with the combination of subjective and objective methods. Suppose there were $n$ unique words among author defined keywords, PubMed MeSH, and key terms extracted from title and abstract of a sample of 30 publications $(p$ $=1,2, \ldots, 30)$. The objective method was as Eq. (2).

$$
\begin{aligned}
& 0 \leq \alpha \leq 1, \text { stepsize }=0.1 \\
& 0 \leq \beta \leq 1-\alpha, \text { stepsize }=0.1 \\
& \gamma=1-\alpha-\beta \\
& \left\{F_{w_{i}, \alpha, \beta, \gamma}^{O}=\alpha f_{1 w_{i}}+\beta f_{2 w_{i}}+\gamma f_{3 w_{i}}, i=1,2, \ldots, n\right. \\
& F_{\alpha, \beta, \gamma}^{O}=\left\{F_{w_{i}, \alpha, \beta, \gamma}^{O}, i=1,2, \ldots, n\right\} \\
& R_{\alpha, \beta, \gamma}^{O}=\operatorname{rank}\left(F_{\alpha, \beta, \gamma}^{O}\right) \\
& R_{\alpha, \beta, \gamma}^{O}=\left\{R_{w_{i}, \alpha, \beta, \gamma}^{O}, i=1,2, \ldots, n\right\}
\end{aligned}
$$

where $\alpha, \beta$, and $\gamma$ represented weights for author defined keywords and PubMed MeSH, key terms extracted from title, and key terms extracted from abstract, respectively. $f_{1 w_{i}}, f_{2 w_{i}}$, and $f_{3 w_{i}}$ represented the frequencies of word $w_{i}$ in author defined keywords and PubMed MeSH, key terms extracted from title, and key terms extracted from abstract, respectively. $F_{w_{i}, \alpha, \beta, \gamma}^{O}$ was the frequency of word $w_{i}$ weighted by $\alpha, \beta$, and $\gamma . F_{\alpha, \beta, \gamma}^{O}$ was the mathematical set of $F_{w_{i}, \alpha, \beta, \gamma}^{O}$. $R_{\alpha, \beta, \gamma}^{O}$ was the objective ranking of $F_{\alpha, \beta, \gamma}^{O}$. $R_{w_{i}, \alpha, \beta, \gamma}^{O}$ was the ranking of word $w_{i}$, and thus $R_{\alpha, \beta, \gamma}^{O}$ was the mathematical set of $R_{w_{i}, \alpha, \beta, \gamma}^{O}$. According to the equation, the total number of $R_{\alpha, \beta, \gamma}^{O}$ was 66 , with 66 kinds of unique combinations of the three parameters.

The subjective method was expressed as Eq. (3).

$$
R_{w_{i}}^{S}=\frac{\sum_{p=1}^{30} R_{p, w_{i}}}{T_{i}}
$$

where $R_{p, w_{i}}$ represented the importance ranking of word $w_{i}$ in sample $p$ and was determined according to specific sample content. If word $w_{i}$ did not appear in sample $p$, then $R_{p, w_{i}}=0 . T_{i}$ was the number of sample containing word $w_{i} . \quad R_{w_{i}}^{S}$ was the average importance ranking of word $w_{i}$.

The optimized combination of the three parameters was determined as Eq. (4).

$$
\left\{\begin{array}{c}
\operatorname{delt}_{\alpha, \beta, \gamma}=\sum_{i=1}^{n}\left|R_{w_{i}}^{S}-R_{w_{i}, \alpha, \beta, \gamma}^{O}\right| \\
\operatorname{delt}_{\text {best }}=\min \left(\operatorname{delt}_{\alpha, \beta, \gamma}\right)
\end{array}\right.
$$

where delt $_{\alpha, \beta, \gamma}$ was the sum of absolute values of the difference between $R_{w_{i}}^{S}$ and $R_{w_{i}, \alpha, \beta, \gamma}^{O}$. delt $t_{b e s t}$ was the minimum of delt $_{\alpha, \beta, \gamma}$.

Using the above method, we got the best combination with $\alpha=0.4, \beta=0.4$, and $\gamma=0.2$.

\section{Affinity propagation clustering analysis}

Affinity Propagation (AP) clustering algorithm based on message passing was proposed by Frey and Dueck [55]. Unlike clustering algorithms such as k-means or kmedoids, AP does not require the setting of cluster numbers in advance. Instead, it simultaneously considers all data points as potential exemplars and recursively transmits real-valued messages until a high-quality set of exemplars and corresponding clusters emerges [56]. For each node $i$ and each candidate exemplar $k$, AP calculates the "responsibility" $r(i, k)$ indicating the suitableness of $k$ as an exemplar for $i$, and the "availability" $a(i$, $k$ ) reflecting the evidence that $i$ should choose $k$ as an exemplar.

$$
r(i, k) \leftarrow s(i, k)-\max _{k^{\prime}: k^{\prime} \neq k}\left\{a\left(i, k^{\prime}\right)+s\left(i, k^{\prime}\right)\right\}
$$




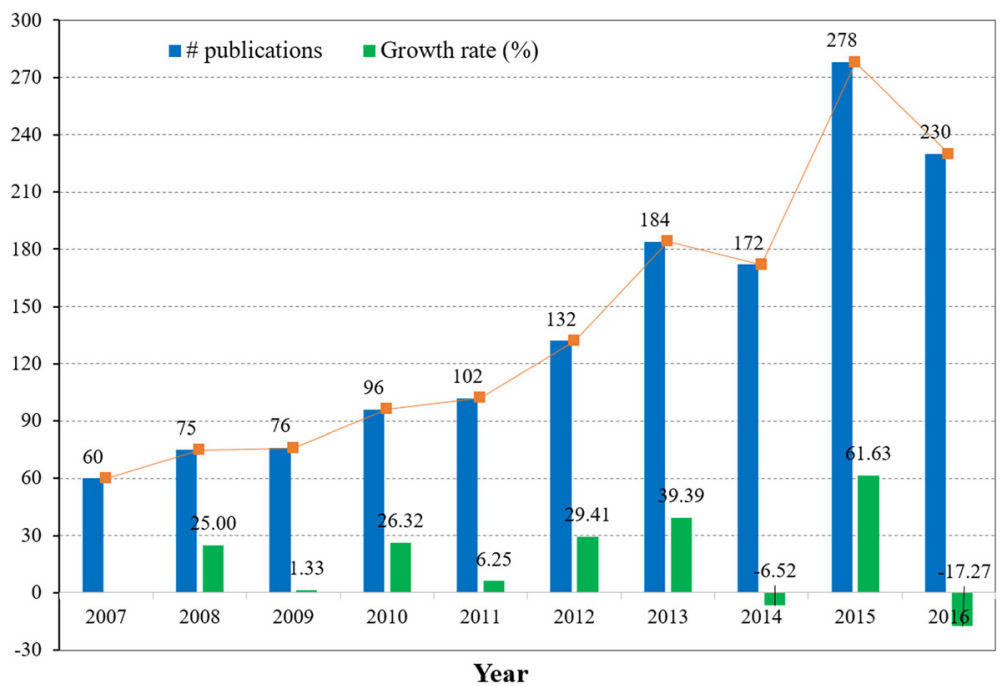

Fig. 1 The number and growth rate of publications by year

$$
a(i, k) \leftarrow \min \left\{0, r(k, k)+\sum_{i^{\prime}: i^{\prime} \notin\{i, k\}} \max \left\{0, r\left(i^{\prime}, k\right)\right\}\right\}
$$

where the matrix $s(i, k)$ indicates the similarities (e.g., edge weights) between two nodes $i$ and $k$, and the diagonal of this matrix contains the preferences for each node. Equations (5) and (6) are iterated until a good set of exemplars emerges. Each node $i$ can then be assigned to the exemplar $k$ which maximizes the sum $a(i, k)+r(i$, $k)$. If $i=k$, then $i$ is an exemplar. A damping factor between 0 and 1 is used to control numerical oscillations.

As reported in literature, AP achieves considerable improvement over standard clustering methods such as k-means [57], spectral clustering [58] and superparamagnetic clustering [59]. It identifies clusters with lower error rate and lower time consumption [60].

We performed AP clustering using an $\mathrm{R}$ package APCluster [61] with a key terms correlation matrix as input data. The matrix was generated based on the cooccurrence matrix using Ochiai correlation coefficient calculated as $O_{i j}=A_{i j} / \sqrt{A_{i} A_{j}} . A_{i}$ and $A_{j}$ represent the frequencies of key terms $W_{i}$ and $W_{j}$, respectively. $A_{i j}$ donates the co-occurrence frequencies of $W_{i}$ and $W_{j}$. The Ochiai coefficient is identical to cosine coefficient [62], thus it can express the similarity of two key terms in theme representation. The value range of $O_{i j}$ is $[0,1]$. The larger the $O_{i j}$ is between two terms, the more similar the two terms are in theme representation.

\section{Results}

Literature distribution characteristics analysis

The number and growth rate of publications by year are shown in Fig. 1. From the figure, the number of NLPempowered medical research publications was overall showing an increasing trend. Until 2012 the number of publications was around 100 per year. From 2013 to 2015, the number of publications increased to around 200 per year. The annual growth rate reached $18.39 \%$ on average, while the rate reached up to $61.63 \%$ from 2014 to 2015, witnessing the research upsurge in 2015. According to regression analysis, the non-linear model with the smallest AIC and biggest $\overline{R^{2}}$ (Table 2) was selected out as $y=6.422397 * 10^{6}-6.408129 * 10^{6} x+1.598485 * 10^{6}$ $\mathrm{x}^{2}$. With this model, the future research output can be estimated.

The 1405 publications were published in 324 unique sources. Table 3 shows the most productive 10 publication sources. These 10 sources together contributed more than $50 \%$ of the total publications. Among them, 8 belonged to journals, i.e., Journal of the American Medical Informatics Association, Journal of Biomedical Informatics, BMC Bioinformatics, PloS ONE, Journal of Biomedical Semantics, Studies in Health Technology and Informatics, BMC Medical Informatics and Decision Making, and Biomedical Informatics Insights. The rest 2 were conferences, i.e., AMIA Annual Symposium Proceedings and AMIA Joint Summits on Translational Science Proceedings.

There were 1386 publications with author affiliation information. The country distribution for first author

Table 2 AIC and $\overline{R^{2}}$ of 3 fitting models

\begin{tabular}{lll}
\hline Model & AlC & $\overline{R^{2}}$ \\
\hline$y=0.06989 x$ & 117.1439 & 0.7829 \\
$y=-45,270.64+22.58 x$ & 98.70681 & 0.855 \\
$y=6.422397^{*} 10^{6}-6.408129^{*} 10^{6} x+1.598485^{*} 10^{6} x^{2}$ & 98.26147 & 0.8703 \\
\hline
\end{tabular}


Table $\mathbf{3}$ Top 10 most productive publication sources

\begin{tabular}{llll}
\hline Publication sources & \# related pub. & $\begin{array}{l}\text { Proportion of related pub. } \\
\text { against 1405 pub. (\%) }\end{array}$ & $\begin{array}{l}\text { Total \#pub. of the sources (Proportion } \\
\text { of related pub. against total \#pub.) }\end{array}$ \\
\hline Journal of the American Medical Informatics Association & 154 & 10.96 & $1689(9.12 \%)$ \\
AMIA Annual Symposium Proceedings & 153 & 10.89 & $2283(6.70 \%)$ \\
Journal of Biomedical Informatics & 133 & 9.47 & $1378(9.65 \%)$ \\
Studies in Health Technology and Informatics & 91 & 6.48 & $7434(1.22 \%)$ \\
BMC Bioinformatics & 61 & 4.34 & $6332(0.96 \%)$ \\
PloS ONE & 36 & 2.56 & $166,876(0.02 \%)$ \\
AMIA Joint Summits on Translational Science Proceedings & 32 & 2.28 & $331(9.67 \%)$ \\
Journal of Biomedical Semantics & 28 & 1.99 & $322(8.70 \%)$ \\
BMC Medical Informatics and Decision Making & 27 & 1.92 & $1071(2.52 \%)$ \\
Biomedical Informatics Insights & 22 & 1.57 & $59(37.29 \%)$ \\
Total & 737 & 52.46 & N/A
\end{tabular}

affiliation was analyzed based on these publications. Table 4 shows the top 8 countries with the highest number of publications and GDP per capita. The USA and Australia were listed in top 8 for the two metrics. According to the Spearman's rank correlation test applied to explore the relationship between publication numbers and GDP per capita, the testing $p$-value was 0.003 , rejecting the null hypothesis at the significance level of $5 \%$. And the Spearman's rank correlation coefficient was 0.445 .

Figure 2 is the Google geomap of country's publications (access via the link [63]). A geomap is a map of a country or continent, with colors and values assigned to specific regions. Values are displayed as a color scale. Here the more publications one country had, the closer the color was to red. For the USA, the red region took a proportion up to $67.17 \%$.

The top productive authors and first authors are presented in Table 5, where $X u$, Hua, Denny, Joshua $C$ and Liu, Hongfang were top 3 productive authors. The top 3 productive first authors were Denny, Joshua $C, X u, H u a$ and Uzuner, Ozlem. Three authors Denny, Joshua $C, X u$, Hua and Uzuner, Ozlem appeared in both two ranks. The top productive author affiliations and first author affiliations are shown in Table 6, where Mayo Clinic, The
University of Utah, and Vanderbilt University ranked top 3 in both ranks.

\section{Scientific collaboration analysis}

The result of publication cooperation analysis on the 1386 publications is shown in Table 7. The number of co-author publications was 1318 during the year 20072016 with an annual co-author rate around 90\%. The co-affiliation rate was generally increasing. Until 2013 the co-affiliation rate was around $45 \%$ per year. From 2014 until 2016, the co-affiliation rate increased to above $60 \%$. The annual co-country rate during 2007-2014 was between $6.38 \%$ and $13.33 \%$, and the number reached up to around $16 \%$ in 2015 and 2016.

We then visualized the cooperation among authors and affiliations. Fig. 3 is a generated network containing 87 authors with publications $>=8$ (access via the link [64]). Fig. 4 shows a force directed network containing 50 affiliations with publications $>=10$ (access via the link [65]). Furthermore, cooperation networks containing 204 authors with publications $>=5$, and 108 affiliations with publications $>=5$, as well as all authors and affiliations were also visualized (access via the link [66-69]). One can interactively drag and drop any

Table 4 Publications and GDP per capita by country

\begin{tabular}{lllll}
\hline Country & \#pub. & Proportion & Country & GDP per capita (1000 US dollars) \\
\hline United States & 931 & $67.17 \%$ & Norway & 897.046 \\
United Kingdom & 72 & $5.19 \%$ & Switzerland & 780.731 \\
China (including Hong Kong and Macao) & 54 & $3.90 \%$ & Denmark & 589.324 \\
France & 50 & $3.61 \%$ & Ireland & 554.754 \\
Canada & 29 & $2.09 \%$ & Australia & 551.685 \\
Germany & 28 & $2.02 \%$ & Sweden & 545.730 \\
Japan & 24 & $1.73 \%$ & United States & 514.139 \\
Australia & 23 & $1.66 \%$ & Netherlands & 506.744 \\
\hline
\end{tabular}




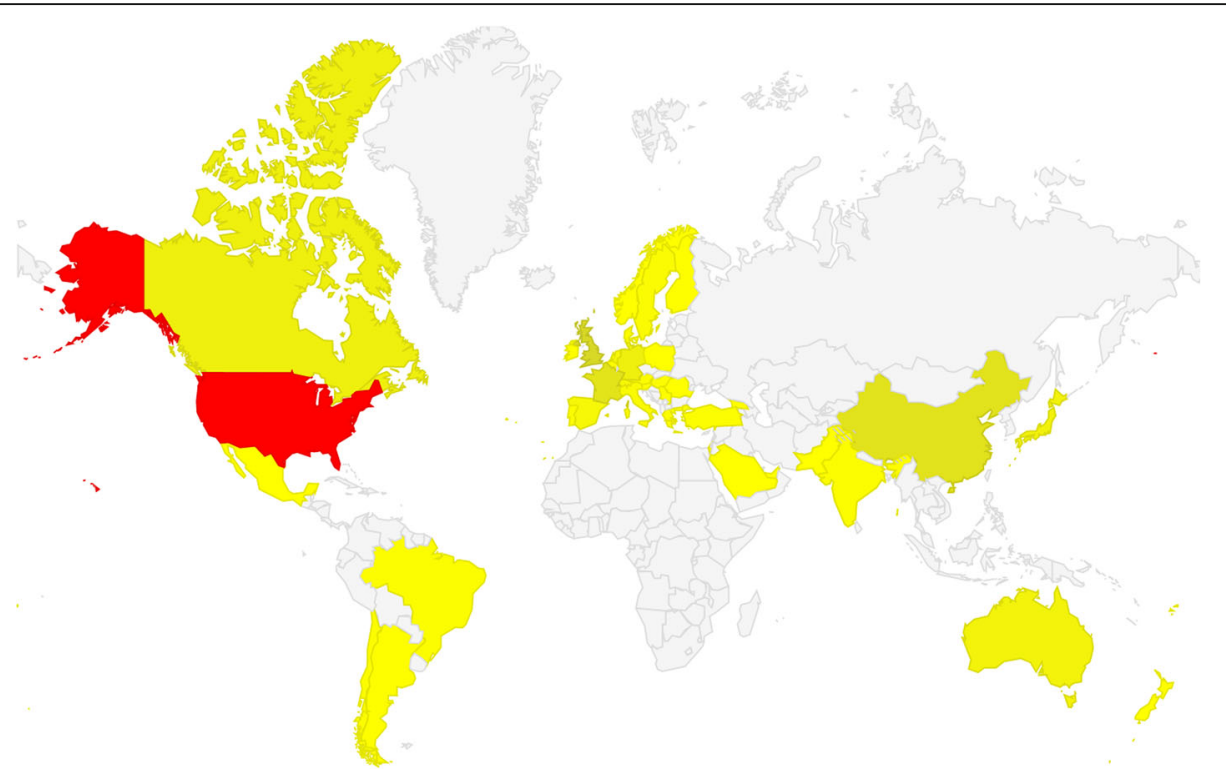

Fig. 2 Geomap visualization of publications by country (the more publications one country had, the closer the color was to red)

node in the networks to view connections for any specific author or affiliation.

\section{Thematic discovery and evolution analysis}

Using the optimized weights combination as $\alpha=0.4$, $\beta=0.4$, and $\gamma=0.2$, we finally obtained top $50 \mathrm{key}$ terms with highest frequencies. Based on these key terms, a 50*50 co-occurrence matrix was generated, where the top 10 are shown in Table 8. The values on the main diagonal of the matrix donated the frequencies of terms and the values on the non-main diagonal indicated the numbers of publications that two terms appeared together.

The correlation matrix generated using Ochiai correlation coefficient was then used for AP clustering. The clustering result for the publication during the year

Table 5 Top productive authors and first authors

\begin{tabular}{llllll}
\hline Rank & Authors & \#pub. & Rank & First authors & \#pub. \\
\hline 1 & Xu, Hua & 54 & 1 & Denny, Joshua C & 12 \\
2 & Denny, Joshua C & 50 & 2 & Xu, Hua & 9 \\
3 & Liu, Hongfang & 41 & 3 & Uzuner, Ozlem & 8 \\
4 & Chute, Christopher G & 27 & 4 & Lacson, Ronilda & 7 \\
5 & Chapman, Wendy W & 25 & 4 & Roberts, Kirk & 7 \\
6 & Friedman, Carol & 24 & 6 & Deleger, Louise & 6 \\
7 & Uzuner, Ozlem & 21 & 6 & Doan, Son & 6 \\
8 & Savova, Guergana K & 20 & 6 & Fan, Jung-Wei & 6 \\
9 & Solti, Imre & 19 & 6 & Gundlapalli, Adi V & 6 \\
10 & Melton, Genevieve B & 18 & 6 & Meystre, Stephane M & 6 \\
10 & Shen, Shuying & 18 & & & \\
10 & Sohn, Sunghwan & 18 & & & \\
\hline
\end{tabular}

2007-2016 was as Fig. 5 and Table 9. The top 50 key terms were distributed into 10 clusters. We manually labelled each cluster by analyzing the meaning of representative terms and reviewing abstract content.

We further compared theme distribution for periods 2007-2011 and 2012-2016, their AP clustering results is shown in Table 10. As for the two periods, top 50 terms were clustered into 12 clusters. Clusters with same exemplars (i.e., cluster 1-8) were placed in top rows. Terms in bold type donated newly emerging terms for 2012-2016 period comparing with 2007-2011.

\section{Discussion}

A bibliometric analysis of NLP-empowered medical research publications from PubMed during the year 20072016 has been conducted. The analysis included three aspects: literature distribution characteristics analysis, scientific collaboration analysis, and thematic discovery and evolution analysis. Some findings were as follows:

1) The NLP-empowered medical research field has attracted the interests of scientific research community throughout years, which was observed in the annual growth of publications.

2) 10 most productive publication sources together contributed more than $50 \%$ of the 1405 publications. The top 3 were: Journal of the American Medical Informatics Association, AMIA Annual Symposium Proceedings, and Journal of Biomedical Informatics.

3) The USA had the highest number of publications with a proportion up to $67.17 \%$. A moderately 
Table 6 Top productive author affiliations and first author affiliations

\begin{tabular}{llllll}
\hline Rank & Author affiliations & \#pub. & Rank & First author affiliations & \#pub. \\
\hline 1 & Mayo Clinic & 86 & 1 & Mayo Clinic & 56 \\
2 & The University of Utah & 82 & 2 & The University of Utah & Vanderbilt University \\
3 & Vanderbilt University & 78 & 3 & Columbia University & 51 \\
4 & National Institutes of Health & 64 & 4 & National Institutes of Health \\
5 & Columbia University & 59 & 5 & Brigham and Women's Hospital \\
6 & Brigham and Women's Hospital & 52 & 6 & University of Minnesota \\
7 & University of Washington & 36 & 7 & University of Pittsburgh \\
8 & University of Pittsburgh & 32 & 7 & VA Salt Lake City Health Care System \\
9 & Massachusetts General Hospital & 31 & 9 & Massachusetts General Hospital & 23 \\
9 & Stanford University & 31 & 10 & 23
\end{tabular}

significant correlation between country's

publications and GDP per capita was revealed by the Spearman's rank correlation coefficient as 0.445 .

4) We have identified prominent authors that have made significant contributions to the research field. Top productive authors included Denny, Joshua $C$, Xu, Hua, Uzuner, Ozlem, and Liu, Hongfang.

5) The top 3 most productive affiliations including Mayo Clinic, The University of Utah, and Vanderbilt University have devoted $17.75 \%$ of the 1386 publications.

6) The annual co-affiliation rate increased to above $60 \%$ from 2014 until 2016, and the annual co-country rate reached up to around 16\% in 2015 and 2016. The cooperation among specific authors and affiliations were visualized using network graphs.

7) The NLP-empowered medical research focused on 10 main thematic areas during the year 2007-2016 including Computational biology, Terminology mining, Information extraction, Text classification, Social medium as data source, Clinical information, Patient characteristics, Performance measurements, Outcome evaluation, and Information retrieval.
8) By observing the newly emerging terms in Table 10, some differences and new research topics can be identified for recent research during the year 20122016 compared with 2007-2011. Especially, cluster $1,2,8,9,10,11$, and 12 were ought to be paid attention to. For example, Information extraction and Named entity recognition have become more popular in medical research during the year 20122016. For Cluster 2, terms related to age, i.e., Middle aged, Adult, and Aged, indicated that researchers gradually paid more attention to the age characteristic of target population in addition to gender. In cluster 8 for 2012-2016 period, the new term Social medium indicated a focus on utilizing social media data for medical analysis [70]. The new term Machine learning in cluster 9 for 2012-2016 period witnessed increasing interest in combining Machine learning and NLP techniques, e.g., [71, 72].

The findings can potentially benefit relevant researchers, especially newcomers in: understanding the research performance and recent development of NLP-empowered medical research field, selecting scientific cooperation

Table 7 The statistics of author and affiliation cooperation

\begin{tabular}{|c|c|c|c|c|c|c|c|}
\hline Year & Total \#pub. & \#co-author pub. & Co-author rate\% & \#co-affiliation pub. & Co-affiliation rate\% & \#co-country pub. & Co-country rate $\%$ \\
\hline 2007 & 58 & 54 & 93.10 & 26 & 44.83 & 7 & 12.07 \\
\hline 2008 & 73 & 64 & 87.67 & 32 & 43.84 & 8 & 10.96 \\
\hline 2009 & 75 & 70 & 93.33 & 36 & 48.00 & 9 & 12.00 \\
\hline 2010 & 94 & 85 & 90.43 & 44 & 46.81 & 6 & 6.38 \\
\hline 2011 & 100 & 96 & 96.00 & 46 & 46.00 & 10 & 10.00 \\
\hline 2012 & 129 & 121 & 93.80 & 63 & 48.84 & 13 & 10.08 \\
\hline 2013 & 180 & 175 & 97.22 & 111 & 61.67 & 24 & 13.33 \\
\hline 2014 & 171 & 161 & 94.15 & 111 & 64.91 & 22 & 12.87 \\
\hline 2015 & 278 & 273 & 98.20 & 170 & 61.15 & 46 & 16.55 \\
\hline 2016 & 228 & 219 & 96.05 & 146 & 64.04 & 36 & 15.79 \\
\hline Total & 1386 & 1318 & N/A & 785 & N/A & 181 & N/A \\
\hline
\end{tabular}




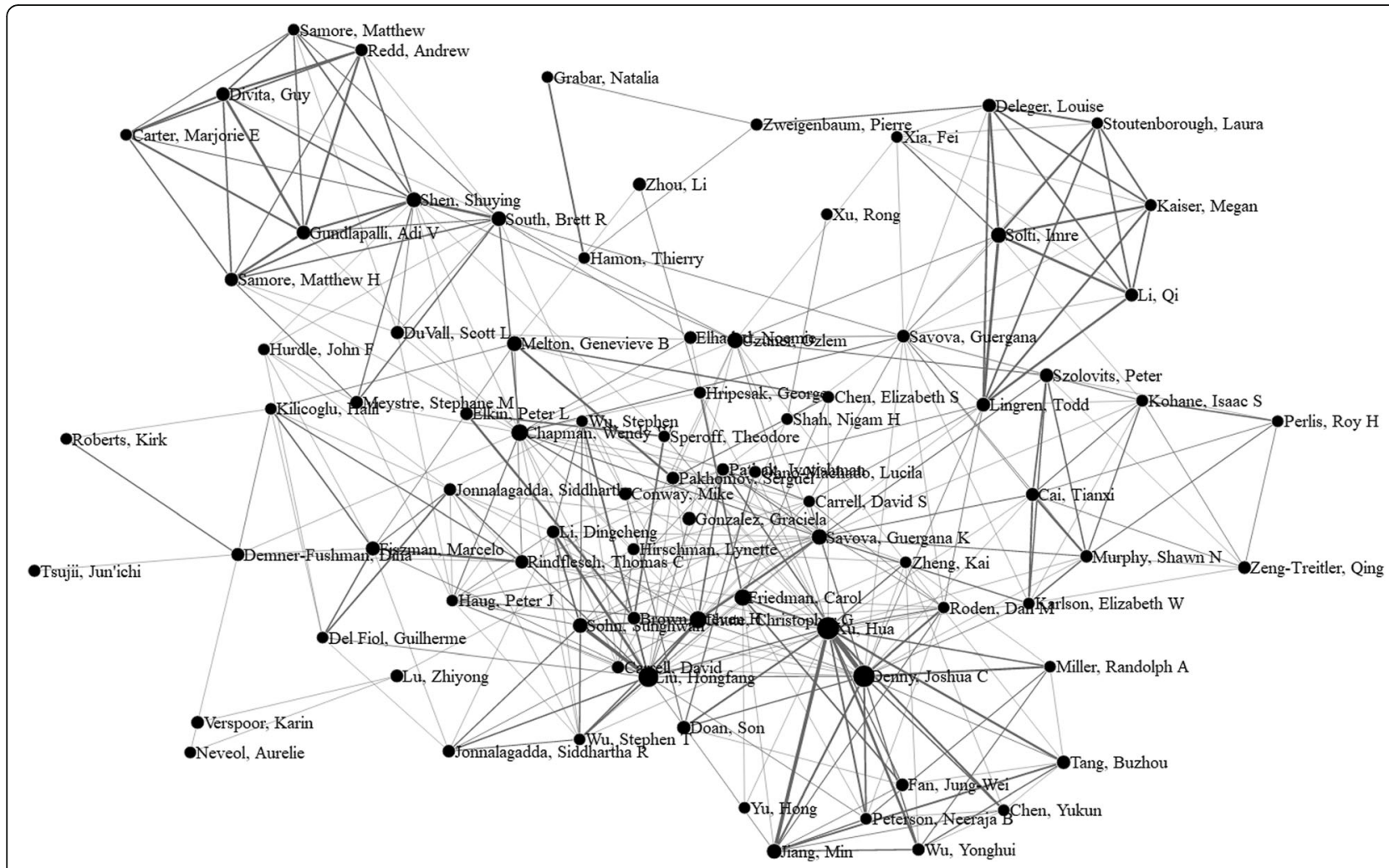

Fig. 3 Force directed network of 87 authors with \#pub. $>=8$

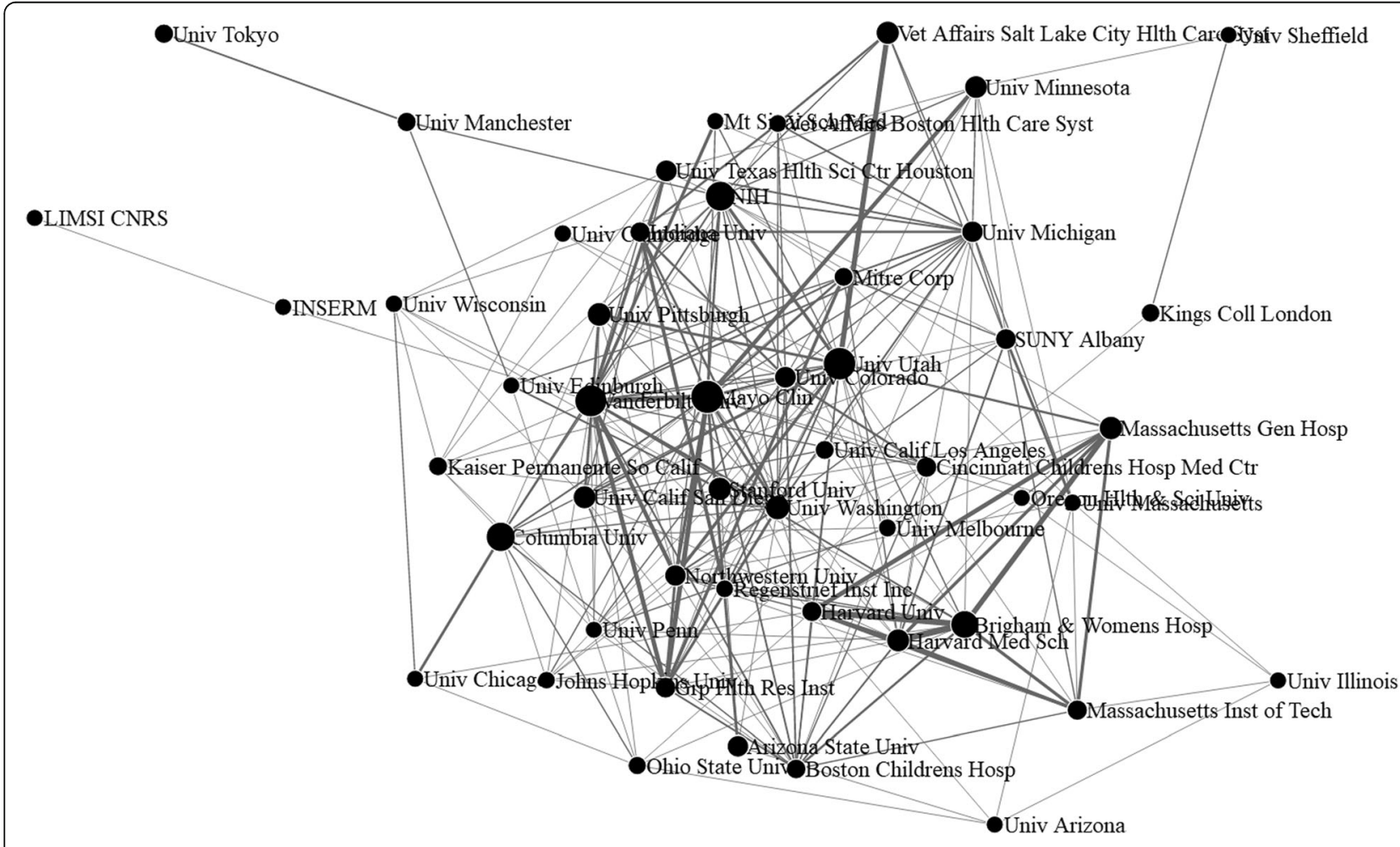

Fig. 4 Force directed network of 50 affiliations with \#pub. $>=10$ 
Table 8 The top 10 key terms in the co-occurrence matrix

\begin{tabular}{|c|c|c|c|c|c|c|c|c|c|c|}
\hline & $\begin{array}{l}\text { Artificial } \\
\text { intelligence }\end{array}$ & $\begin{array}{l}\text { Data } \\
\text { mining }\end{array}$ & $\begin{array}{l}\text { Electronic health } \\
\text { record }\end{array}$ & Female & $\begin{array}{l}\text { Information storage } \\
\text { and retrieval }\end{array}$ & $\begin{array}{l}\text { Machine } \\
\text { learning }\end{array}$ & $\begin{array}{l}\text { Medical } \\
\text { record }\end{array}$ & Patient & Precision & Semantics \\
\hline Artificial intelligence & 185 & 52 & 53 & 11 & 56 & 40 & 25 & 33 & 40 & 33 \\
\hline Data mining & 52 & 288 & 122 & 31 & 20 & 53 & 38 & 55 & 46 & 52 \\
\hline Electronic health record & 53 & 122 & 420 & 78 & 80 & 60 & 95 & 167 & 77 & 40 \\
\hline Female & 11 & 31 & 78 & 169 & 15 & 10 & 46 & 82 & 18 & 10 \\
\hline $\begin{array}{l}\text { Information storage and } \\
\text { retrieval }\end{array}$ & 56 & 20 & 80 & 15 & 239 & 18 & 30 & 42 & 47 & 47 \\
\hline Machine learning & 40 & 53 & 60 & 10 & 18 & 162 & 25 & 39 & 30 & 22 \\
\hline Medical record & 25 & 38 & 95 & 46 & 30 & 25 & 178 & 77 & 29 & 8 \\
\hline Patient & 33 & 55 & 167 & 82 & 42 & 39 & 77 & 326 & 59 & 19 \\
\hline Precision & 40 & 46 & 77 & 18 & 47 & 30 & 29 & 59 & 217 & 34 \\
\hline Semantics & 33 & 52 & 40 & 10 & 47 & 22 & 8 & 19 & 34 & 165 \\
\hline
\end{tabular}

partners with the knowledge of predominant authors, affiliations, and countries, optimizing research topic decision to keep abreast of current research hotspots, and monitoring new scientific or technological activities.

In term importance weighting, the combination of subjective and objective methods was used. The subjective weighing result might vary from person to person due to subjective judgment. Thus, in this paper, we ranked the importance by semantics analysis as well as reviewing text content to keep high consistence with text intention.

In our study, AP clustering method was performed based on top 50 high frequency key terms in order to acquire a moderate number of categories. However, this might result in the ignorance of some sudden terms that are possible for representing research fronts although with low frequencies. Therefore, in our future work, we will make improvement by trying alternative methods such as Latent Dirichlet Allocation to consider every single term.

The AP clustering results were on the whole reasonable and easy-to-understand. However, we still found that terms with similar semantics, i.e., Data mining and Text mining were not clustered into the same cluster in the same context NLP, which might cause confusion. AP clustering was conducted based on the key terms correlation matrix, and the matrix was calculated using Ochiai

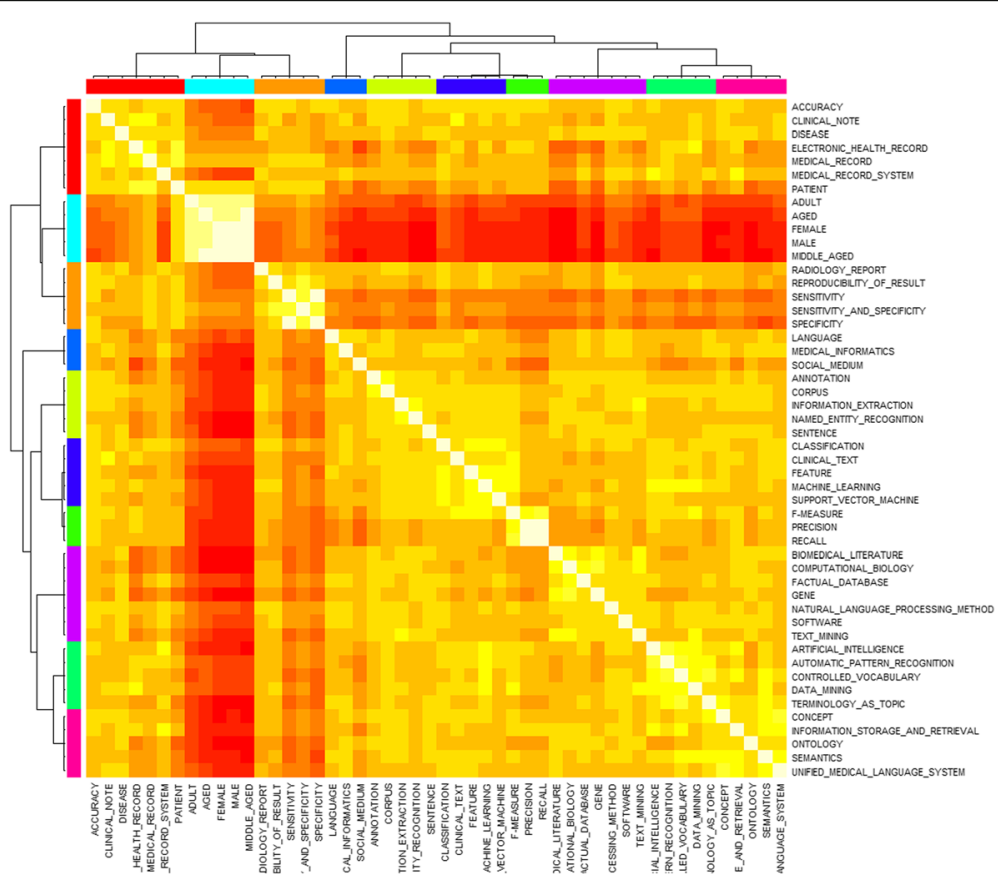

Fig. 5 Heatmap of AP clustering result for the 2007-2016 period 
Table 9 AP clustering result for the publication during the year2007-2016

\begin{tabular}{|c|c|c|}
\hline Cluster & Theme & Key terms \\
\hline 1 & Computational biology & $\begin{array}{l}\text { Computational biology; Biomedical literature; Factual database; Gene; Natural language } \\
\text { processing method; Software; Text mining }\end{array}$ \\
\hline 2 & Terminology mining & $\begin{array}{l}\text { Controlled vocabulary; Artificial intelligence; Automatic pattern recognition; Data mining; } \\
\text { Terminology as topic }\end{array}$ \\
\hline 3 & Information extraction & Corpus; Annotation; Information extraction; Named entity recognition; Sentence \\
\hline 4 & Text classification & Feature; Classification; Clinical text; Machine learning; Support vector machine \\
\hline 5 & Social medium as data source & Language; Medical informatics; Social medium \\
\hline 6 & Clinical information & $\begin{array}{l}\text { Medical record; Accuracy; Clinical note; Disease; Electronic health record; Medical record } \\
\text { system; Patient }\end{array}$ \\
\hline 7 & Patient characteristics & Middle aged; Adult; Aged; Female; Male \\
\hline 8 & Performance measurements & Recall; F-measure; Precision \\
\hline 9 & Outcome evaluation & Sensitivity and specificity; Radiology report; Reproducibility of result; Sensitivity; Specificity \\
\hline 10 & Information retrieval & Unified medical language system; Concept; Information storage and retrieval; Ontology; Semantics \\
\hline
\end{tabular}

correlation coefficient. Hence, the clustering results might be vulnerable to choices of both calculation method and clustering method. Therefore, in our future work, we will conduct comparison on different calculation methods of correlation matrix as well as different clustering methods for further exploration.

In the study, PubMed as the biggest medical related publication resource was used as data source. However, a minor number of publications might be in NLP-related journals and conferences. Thus, in our future work, we will consider including these journals and conference as additional publication sources.

\section{Conclusions}

This paper presents a bibliometric analysis of NLPempowered medical research publications from PubMed during the year 2007-2016 with the purpose of understanding the research status of the field. Some literature distribution characteristics including productive publication sources, authors, affiliations, and countries are

Table 10 Comparison of AP clustering results for the 2007-2011 and 2012-2016 periods

\begin{tabular}{|c|c|c|c|}
\hline Cluster & $2007-2011$ & Cluster & $2012-2016$ \\
\hline 1 & $\begin{array}{l}\text { Text mining; Abstracting and indexing as topic; Annotation; } \\
\text { Database management system; Sentence }\end{array}$ & 1 & Text mining; Information extraction; Named entity recognition \\
\hline 2 & Female; Male & 2 & Female; Male; Middle aged; Adult; Aged \\
\hline 3 & Recall; Precision; F-measure & 3 & Recall; Precision; F-measure; Accuracy \\
\hline 4 & $\begin{array}{l}\text { Artificial intelligence; Information storage and retrieval; } \\
\text { Automatic pattern recognition }\end{array}$ & 4 & $\begin{array}{l}\text { Artificial intelligence; Semantics; Information storage and retrieval; } \\
\text { Clinical text; Concept; Language; Sentence; Unified medical language } \\
\text { system }\end{array}$ \\
\hline 5 & $\begin{array}{l}\text { Computational biology; Factual database; Gene; Protein; } \\
\text { Protein-protein interaction }\end{array}$ & 5 & Computational biology; Factual database; Software; Free text \\
\hline 6 & $\begin{array}{l}\text { Classification; Feature; Semantics; Data mining; Natural } \\
\text { language processing method; Unified medical language } \\
\text { system }\end{array}$ & 6 & Classification; Feature; Support vector machine; Classifier \\
\hline 7 & $\begin{array}{l}\text { Patient; Disease; Medical record; Medical record system; } \\
\text { Patient discharge; Sensitivity and specificity }\end{array}$ & 7 & Patient; Medical record; Electronic health record; Clinical note \\
\hline 8 & Medical informatics; User-computer interface; Software & 8 & Medical informatics; Annotation; Corpus; Gene; Social medium \\
\hline 9 & $\begin{array}{l}\text { Clinical text; Accuracy; Clinical decision support system; } \\
\text { Clinical note; Electronic health record; Natural language } \\
\text { processing system; Support vector machine }\end{array}$ & 9 & $\begin{array}{l}\text { Automatic pattern recognition; Controlled vocabulary; Data mining; } \\
\text { Machine learning }\end{array}$ \\
\hline 10 & Word; Corpus; Language & 10 & Sensitivity; Confidence interval; Specificity \\
\hline 11 & Biomedical literature; Knowledge; Medline; Ontology & 11 & $\begin{array}{l}\text { Reproducibility of result; Radiology report; Sensitivity and } \\
\text { specificity }\end{array}$ \\
\hline 12 & Terminology as topic; Concept; Controlled vocabulary & 12 & Disease; Natural language processing method; Phenotype \\
\hline
\end{tabular}

First term in each cluster donates exemplar. Terms in bold type donate new emergent terms for 2012-2016 period compared with $2007-2011$ period 
provided with statistics analysis methods. Scientific collaborations among authors and affiliations are visualized with network analysis method. Affinity propagation clustering method is used for thematic discovery and evolution analysis. Some interesting results and findings are presented. To our knowledge, there was no similar study thoroughly examining NLP-empowered medical research publications. Our work can potentially assist relevant researchers, especially newcomers in keeping abreast of the NLP-empowered medical research status, seeking scientific cooperation partners, optimizing research topics choices, and monitoring new scientific or technological activities.

\section{Abbreviations}

AIC: Akaike Information Criterion; AP: Affinity Propagation; EHR: Electronic Health Record; EHRs: Electronic Health Records; EMRs: Electronic Medical Records; GDP: Gross Domestic Product; MeSH: Medical subject headings; $\mathrm{NIH}$ : National Institutes of Health; NLP: Natural Language Processing; USA: United States; WoS: Web of Science

\section{Funding}

Publication of the article is supported by grants from National Natura Science Foundation of China (No.61772146), Research Grants Council of Hong Kong Special Administrative Region, China (UGC/FDS11/E04/16), and Innovative School Project in Higher Education of Guangdong Province (No.YQ2015062)

\section{Availability of data and materials}

The datasets used and analyzed during the current study are available from the corresponding author upon reasonable requests.

\section{About this supplement}

This article has been published as part of BMC Medical Informatics and Decision Making Volume 18 Supplement 1, 2018: Proceedings from the 3rd China Health Information Processing Conference (CHIP 2017). The full contents of the supplement are available online at https://bmcmedinformdecismak.biomedcentral.com/articles/ supplements/volume-17-supplement-1.

\section{Authors' contributions}

$\mathrm{XLC}$ leaded the method application, experiment conduction and the result analysis. HRX, FLW, ZQL, and JX participated in the design of the research and the revision of the manuscript. TYH provided theoretical guidance, the key term extraction program development and the revision of this paper. All authors read and approved the final manuscript.

\section{Ethics approval and consent to participate} Not applicable.

\section{Consent for publication}

Not applicable.

\section{Competing interests}

The authors declare that they have no competing interests.

\section{Publisher's Note}

Springer Nature remains neutral with regard to jurisdictional claims in published maps and institutional affiliations.

\footnotetext{
Author details

${ }^{1}$ College of Economics, Jinan University, Guangzhou, China. ${ }^{2}$ Department of Mathematics and Information Technology, The Education University of Hong Kong, Hong Kong, Hong Kong, Special Administrative Region of China. ${ }^{3}$ School of Science and Technology, The Open University of Hong Kong, Hong Kong, Hong Kong, Special Administrative Region of China. ${ }^{4}$ The Second Clinical Medical College, Guangzhou University of Chinese Medicine, Guangzhou, China. ${ }^{5}$ The Research Institute of National Supervision and Audit
}

Law, Nanjing Audit University, Nanjing, China. ${ }^{6}$ School of Information Science and Technology, Guangdong University of Foreign Studies, Guangzhou, China. ${ }^{7}$ School of Computer, South China Normal University, Guangzhou, China.

Published: 22 March 2018

\section{References}

1. Cambria $E$, White $B$. Jumping NLP curves: a review of natural language processing research. IEEE Comput Intell Mag. 2014;9(2):48-57.

2. Liddy ED. Natural language processing. In: Encyclopedia of Library and Information Science. New York: 2nd ed; 2001. p. 2126-36.

3. Batet M, Sánchez D, Valls A. An ontology-based measure to compute semantic similarity in biomedicine. J Biomed Inform. 2011;44(1):118-25.

4. Meystre S, Automation HPJ. Of a problem list using natural language processing. BMC medical informatics and decision making. 2005;5(1):30.

5. Wang PW, Hao TY, Jin LW, Yan J. Large-Scale Extraction of drug-disease pairs from biomedical literature for drug repurposing. Journal of the Association for Information Science and Technology. 2017;68(11):2649-61.

6. Névéol A, Zweigenbaum P. Clinical natural language processing in 2015: leveraging the variety of texts of clinical interest. IMIA Yearbook. 2016:234-9.

7. Xiao B, Imel ZE, Georgiou PG, Atkins DC, Narayanan SS. "Rate my therapist": automated detection of empathy in drug and alcohol counseling via speech and language processing. PLoS One. 2015;10(12):e0143055.

8. Z Zhang YY, Tang BZ, Jiang M, Wang JQ, Xu H. Domain adaptation for semantic role labeling of clinical text. J Am Med Inform Assoc. 2015;22(5): 967-79.

9. Sidorov G, Velasquez F, Stamatatos E, Gelbukh A, Chanona-Hernández L. Syntactic n-grams as machine learning features for natural language processing. Expert Syst Appl. 2014;41(3):853-60.

10. Chasin R, Rumshisky A, Uzuner O, Szolovits P. Word sense disambiguation in the clinical domain: a comparison of knowledge-rich and knowledge-poor unsupervised methods. J Am Med Inform Assoc. 2014;21(5):842-9.

11. Wang Y, Zheng K, Xu H, Mei QZ. Clinical word sense disambiguation with interactive search and classification. In: Proc. of AMIA annual symposium; 2016. p. 2062-71.

12. Nikfarjam A, Sarker A, O'Connor K, Ginn R, Gonzalez G. Pharmacovigilance from social media: mining adverse drug reaction mentions using sequence labeling with word embedding cluster features. J Am Med Inform Assoc. 2015;22(3):671-81.

13. Sun WY, Rumshisky A, Uzuner O. Normalization of relative and incomplete temporal expressions in clinical narratives. J Am Med Inform Assoc. 2015; 22(5):1001-8.

14. Albers DJ, Elhadad N, Tabak E, Perotte A, Hripcsak G. Dynamical phenotyping: using temporal analysis of clinically collected physiologic data to stratify populations. PLoS One. 2014;9(6):e96443.

15. Lin C, Dligach D, Miller TA, Bethard S, Savova GK. Multilayered temporal modeling for the clinical domain. J Am Med Inform Assoc. 2015;23(2):387-95.

16. Li Q, Spooner SA, Kaiser M, Lingren N, Robbins J, Lingren T, et al. An end-toend hybrid algorithm for automated medication discrepancy detection. BMC medical informatics and decision making. 2015;15(1):37-49.

17. Zheng L, Wang $Y$, Hao SY, Shin AY, Jin B, Ngo AD, et al. Web-based realtime case finding for the population health Management of Patients with Diabetes Mellitus: a prospective validation of the natural language processing-based algorithm with statewide electronic medical records. JMIR medical informatics. 2016;4(4):e37.

18. Hao TY, Rusanov A, Boland MR, Weng CH. Clustering clinical trials with similar eligibility criteria features. J Biomed Inform. 2014;52:112-20.

19. Hao TY, Liu HF, Weng CH. Valx: a system for extracting and structuring numeric lab test comparison statements from text. Methods Inf Med. 2016; 55(3):266-75.

20. Hao TY, Weng $\mathrm{CH}$. Adaptive Semantic tag mining from heterogeneous clinical research texts. Methods Inf Med. 2015:54(2):164-70.

21. Hao TY, Chen XL, Huang GM. Discovering commonly shared semantic concepts of eligibility criteria for learning clinical trial design. Lect Notes Comput Sci. 2015;9412:3-13.

22. Cobo MJ, Martínez MA, Gutiérrez-Salcedo M, Fujita H, Herrera-Viedma E. 25 years at knowledge-based systems: a bibliometric analysis. Knowl-Based Syst. 2015;80:3-13.

23. Cobo MJ, López-Herrera AG, Herrera-Viedma E, Herrera F. An approach for detecting, quantifying, and visualizing the evolution of a research field: a 
practical application to the fuzzy sets theory field. Journal of Informetrics. 2011;5(1):146-66.

24. Chen XL, Chen BY, Zhang CX, Hao TY. Discovering the recent research in natural language processing field based on a statistical approach. Lect Notes Comput Sci. 2017;10676:507-17.

25. Wallace ML, Larivière V, Gingras Y. A small world of citations? The influence of collaboration networks on citation practices. PLoS One. 2012;7(3):e33339.

26. Chen XL, Weng H, Hao TY. A data-driven approach for discovering the recent research status of diabetes in China. Lect Notes Comput Sci. 2017; 10594:89-101.

27. Boudry C, Mouriaux F. Eye neoplasms research: a bibliometric analysis from 1966 to 2012. Eur J Ophthalmol. 2015;25(4):357-65.

28. Diem A, Wolter SC. The use of bibliometrics to measure research performance in education sciences. Res High Educ. 2013;54(1):86-114.

29. Fan XM, Gao Y, Ma B, Xia ZF. Chinese academic contribution to burns: a comprehensive bibliometrics analysis from 1985 to 2014. Burns. 2016;42(7): 1463-70.

30. Venable GT, Khan NR, Taylor DR, Thompson CJ, Michael LM, Klimo P. A correlation between National Institutes of Health funding and bibliometrics in neurosurgery. World neurosurgery. 2014;81(3):468-72.

31. Harande YI, Alhaji IU. Basic Literature of diabetes: a bibliometrics analysis of three countries in different world regions. Journal of Library and Inf Sci. 2014;2(1):49-56.

32. Ramos JM, Padilla S, Masia M, Gutierrez F. A bibliometric analysis of tuberculosis research indexed in PubMed, 1997-2006. The International Journal of Tuberculosis and Lung Disease. 2008;12(12):1461-8.

33. Holliday EB, Ahmed AA, Yoo SK, Jagsi R, Hoffman KE. Does cancer literature reflect multidisciplinary practice? A systematic review of oncology studies in the medical literature over a 20-year period. Int J Radiat Oncol Biol Phys. 2015;92(4):721-31.

34. Boudry C, Denion E, Mortemousque B, Mouriaux F. Trends and topics in eye disease research in PubMed from 2010 to 2014. PeerJ. 2016:4:e1557.

35. Khan A, Choudhury N, Uddin S, Hossain L, Baur LA. Longitudinal trends in global obesity research and collaboration: a review using bibliometric metadata. Obes Rev. 2016;17(4):377-85.

36. Kramer PF, Onetto J, Flores MT, Borges TS, Feldens CA. Traumatic dental injuries in the primary dentition: a 15-year bibliometric analysis of dental traumatology. Dent Traumatol. 2016;32(5):341-6.

37. Velmurugan C. Research trends in journal of intellectual property rights (JIPR): a bibliometric study. Libr Philos Pract. 2013;1043:1-16.

38. Hussain A, Fatima N, Kumar D. Bibliometric analysis of the'Electronic Librany'journal (2000-2010). Webology. 2011:8(1):87.

39. Newman ME. Coauthorship networks and patterns of scientific collaboration. Proc Natl Acad Sci. 2004;101(1):5200-5.

40. Radev DR, Joseph MT, Gibson B, Muthukrishnan P. A bibliometric and network analysis of the field of computational linguistics. Journal of the Association for Information Science and Technology. 2016;67(3):683-706.

41. Muñoz-Leiva F, Viedma-del-Jesús MI, Sánchez-Fernández J, López-Herrera AG. An application of co-word analysis and bibliometric maps for detecting the most highlighting themes in the consumer behaviour research from a longitudinal perspective. Quality \& Quantity. 2012;46(4):1077-95.

42. Geaney F, Scutaru C, Kelly C, Glynn RW, Perry IJ. Type 2 diabetes research yield, 1951-2012: bibliometrics analysis and density-equalizing mapping. PLoS One. 2015;10(7):e0133009

43. Li L, Liu Y, Zhu HH, Ying S, Luo QY, Luo H, et al. A bibliometric and visual analysis of global geoontology research. Comput Geosci. 2017;99:1-8.

44. Heo GE, Kang KY, Song M, Lee JH. Analyzing the field of bioinformatics with the multifaceted topic modeling technique. BMC bioinformatics. 2017;18(7):251.

45. Jiang HC, Qiang MS, Lin P. A topic modeling based bibliometric exploration of hydropower research. Renew Sust Energ Rev. 2016;57:226-37.

46. Yeung AWK, Goto TK, Leung WK. The changing landscape of neuroscience research, 2006-2015: a bibliometric study. Front Neurosci. 2017;11:120

47. Martínez MA, Cobo MJ, Herrera M, Herrera-Viedma E. Analyzing the scientific evolution of social work using science mapping. Res Soc Work Pract. 2015; 25(2):257-77.

48. Cobo MJ, Chiclana F, Collop A, de Ona J, Herrera-Viedma E. A bibliometric analysis of the intelligent transportation systems research based on science mapping. IEEE Trans Intell Transp Syst. 2014;15(2):901-8.

49. Dehdarirad T, Villarroya A, Barrios M. Research trends in gender differences in higher education and science: a co-word analysis. Scientometrics. 2014; 101(1):273-90.
50. Mann PS. Introductory statistics. New York: John Wiley \& Sons; 2007.

51. MacEachren AM, Boscoe FP, Haug D, Pickle LW. Geographic visualization: designing manipulable maps for exploring temporally varying georeferenced statistics. Proc of IEEE Information Visualization Symposium. 1998:87-94.

52. Otte E, Rousseau R. Social network analysis: a powerful strategy, also for the information sciences. J Inf Sci. 2002;28(6):441-53.

53. Wasserman S, Faust K. Social network analysis: methods and applications. Press: Cambridge Univ; 1994.

54. Serrat O. Social network analysis. Singapore: Knowledge solutions. Springer; 2017. p. 39-43.

55. Frey BJ, Dueck D. Clustering by passing messages between data points. Science. 2007:315(5814):972-6.

56. Frey BJ, Dueck D. Response to comment on "clustering by passing messages between data points". Science. 2008;319(5864):726.

57. MacQueen J. Some methods for classification and analysis of multivariate observations. In: Proc of the fifth Berkeley symposium on mathematical statistics and probability. 1967;1(14):281-97.

58. Shi JB, Malik J. Normalized cuts and image segmentation. IEEE Trans Pattern Anal Mach Intell. 2000;22(8):888-905.

59. Shental N, Zomet A, Hertz T, Weiss Y. Pairwise clustering and graphical models. Adv Neural Inf Proces Syst. 2004:185-92.

60. El-Samak AF, Ashour W. Optimization of traveling salesman problem using affinity propagation clustering and genetic algorithm. Journal of Artificial Intelligence and Soft Computing Research. 2015;5(4):239-45.

61. Bodenhofer U, Kothmeier A, Hochreiter S. APCluster: an R package for affinity propagation clustering. Bioinformatics. 2011;27(17):2463-4.

62. Romesburg C. Cluster analysis for researchers: Lulu Press; 2004.

63. Geomap of country publication. http://www.zhukun.org/haoty/resources. asp?id=BMC_publication_map. Accessed 20 Nov 2017.

64. The network of authors (\#pub.>= 8). http://www.zhukun.org/haoty/resources. asp?id=BMC_coauthor_8 (Accessed 20 Nov 2017).

65. The network of affiliations (\#pub.>=10). http://www.zhukun.org/haoty/ resources.asp?id=BMC_affiliation_10 (Accessed 20 Nov 2017).

66. The network of authors (\#pub.>=5). http://www.zhukun.org/haoty/resources. asp?id=BMC_coauthor_5 (Accessed 20 Nov 2017).

67. The network of affiliations (\#pub.>= 5). http://www.zhukun.org/haoty/ resources.asp?id=BMC_affiliation_5 (Accessed 20 Nov 2017).

68. The network of all authors. http://www.zhukun.org/haoty/resources.asp?id= BMC_coauthor_all (Accessed 20 Nov 2017).

69. The network of all affiliations. http://www.zhukun.org/haoty/resources.asp?id= BMC_affiliation_all (Accessed 20 Nov 2017).

70. Demner-Fushman D, ElhadaXd N. Aspiring to unintended consequences of natural language processing: a review of recent developments in clinical and consumer-generated text processing. IMIA Yearbook. 2016:224-33.

71. Osborne JD, Wyatt M, Westfall AO, Willig J, Bethard S, Gordon G. Efficient identification of nationally mandated reportable cancer cases using natural language processing and machine learning. J Am Med Inform Assoc. 2016; 23(6):1077-84.

72. Yadav K, Sarioglu E, Choi H, Cartwright WB, Hinds PS, Chamberlain JM. Automated outcome classification of computed tomography imaging reports for pediatric traumatic brain injury. Acad Emerg Med. 2016;23(2):171-8.

\section{Submit your next manuscript to BioMed Central and we will help you at every step:}

- We accept pre-submission inquiries

- Our selector tool helps you to find the most relevant journal

- We provide round the clock customer support

- Convenient online submission

- Thorough peer review

- Inclusion in PubMed and all major indexing services

- Maximum visibility for your research

Submit your manuscript at www.biomedcentral.com/submit 